\title{
MiPyme En el Giro Industrial : Factores de Competencia
}

\author{
Dr. Jesús Francisco Mellado Siller \\ Mtra. Edith Reyes Ruiz. \\ Dra. Adriana Méndez Wong
}

Universidad Autónoma de Coahuila, México

Doi: 10.19044/esj.2017.v13n34p80 URL:http://dx.doi.org/10.19044/esj.2017.v13n34p80

\begin{abstract}
The present study aims to determine which of the factors that considers the industrial small and medium enterprises. It makes them feel that they are better than their competitors. For this purpose, a factorial analysis of the variables was applied. These are findings which show that only two are the main factors in the industry. First, it involves what is related to internal marketing in terms of efficient processes, satisfied employees, and companies that have good performance and which are more profitable. Second, it also entails what concerns competitiveness. Here, we have the items of higher quality products, customer satisfaction, and adaptation to market changes that improves their intangible value.
\end{abstract}

Keywords: Performance indicators, internal marketing, competitiveness

\section{Resumen}

El presente trabajo intenta determinar cuáles factores consideran las miPyme de giro industrial, que intervienen para que consideren que están mejor que sus competidores; para ello se aplica un análisis factorial de las variables encontrándose que solo son dos factores los principales en la industria; lo relacionado con la mercadotecnia interna, en cuanto a procesos eficientes, empleados satisfechos, las empresas tienen rendimiento, y son más rentables. Y lo concerniente a la competitividad, donde se tienen los ítems de productos de mayor calidad, satisfacción del cliente y adaptación a los cambios de mercado, mejorando su valor intangible.

Palabras-Clave: Indicadores de Rendimiento, marketing interno, competitividad 


\section{Introducción}

La micro, pequeña y mediana empresa (miPyme) ha recibido en estos años mucha atención, por parte de trabajos de investigación, debido al el papel que están jugando en el mundo entero, se ha demostrado la importancia de este tipo de negocios, en lo que respecta al desarrollo de la economía que se ha tenido en cada uno de los países donde se desarrollan este tipo de empresas, así como la importancia que tienen en cuanto a la generación de empleos.

Las miPyme's quizá no deberían ser llamadas empresas, debido a la carencia que presentan en todos los aspectos de sus fundamentos operativos; una fuerte escasez de herramientas para administrarse, dificultades para obtener capital, manejo de sus aspectos financieros, estrategias de mercado efectivas, estrategias de internacionalización para poder ingresar en el mercado global que actualmente opera en todo el planeta.

Esto es lo que se ha llamado debilidades de carácter estructural (García Pérez de Lerma, Martínez García , \& Aragón Sánchez, 2011) es lo que dificulta las relaciones que tienen con su entorno, lo que se hace necesario reconocer que su estudio es necesario para determinar cuáles son las más importantes de sus características, y como la sociedades gubernamentales tienen que ayudar a encarar, junto con ellas, la problemática de sus funciones y poder intervenir para que estos negocios puedan seguir con un desarrollo de manera constante lo que implicaría para el país una mejora económica y de riqueza.

Las miPyme's están necesitadas de conocimiento acerca de sus fundamentos operativos, sobre todo en lo concerniente al área de la mercadotecnia, en sus políticas de corte nacional e internacional, esto permite un desarrollo lento, afectando la supervivencia además de que su competitividad es totalmente baja, la dificultad de manejo de las nuevas tecnologías realiza más mermas en su desarrollo competitivo e impiden un ingreso en los nuevos mercados. Así, la dificultad para la captación de capitales ajenos a largo plazo, la menor cualificación de empresarios y empleados, las dificultades de localización e infraestructura, y la complejidad para incorporar las innovaciones tecnológicas son, entre otros, aspectos que dificultan y limitan el desarrollo de la miPyme.

\section{Revisión de Literatura}

Los indicadores de rendimiento de las miPyme's, están construidos para medir la percepción del gerente de la empresa con respecto a la posición competitiva, frente a la alternativa de utilizar indicadores procedentes de la información contable, ya que ésta puede verse alterada por la propia normatividad de la contabilidad de la empresa, o por las decisiones 
directivas. Es esta una medida de éxito en tiempo pasado más que del presente o del futuro, Pérez de Lema (2011).

El marco de referencia de la evaluación de competencias son utilizados para examinar las relaciones entre la cultura organizacional y otras variables de las organizaciones, como la satisfacción del trabajo (Lund, 2003) desde un impacto de la cultura organizacional los tipos de la satisfacción en las encuestas profesionales de marketing, en una sección de firmas en Estados Unidos de Norteamérica.

El marco de referencia de la evaluación fue utilizado como un concepto referente para analizar. Los resultados indican que la satisfacción de trabajo fue positivamente relacionada con un grupo social y con falta de jerarquía cultural, y son negativamente relacionadas para el mercado y la jerarquía cultural. Estos estudios empíricos son validados por el marco de referencia de evaluación como un poderoso instrumento para la evaluación de la cultura organizacional, en el ámbito de la competencia.

El mecanismo del mercado, precios, son la información necesaria para una toma de decisión eficiente, debido a que se tienen diferentes mecanismos de precios, la compañía también puede simplemente utilizar a los empleados en una directa proporción para su contribución. Cada una de los mecanismos requiere un sistema de información que puede incluir contadores, expertos en computadoras para evaluar este procedimiento, constituyendo así una manera de obtener una visión acerca del sitio que se ocupa dentro del ámbito empresarial competitivamente.

Este tipo de relaciones entre las etapas de desarrollo de los ciclos de vida en una organización y los cuatro tipos de cultura en el cvf. Quinn y Cameron (1983) revisaron los nueve modelos de los ciclos de vida en una organización y ellos propusieron en la literatura un modelo derivado de cada uno de los integrantes en cada uno de los nueve modelos. El ciclo de vida del modelo incluye cuatro etapas: gerencial, colectividad, formalización y control y la elaboración de la estructura. Está basado con ciertas características del tipo de empresa en diferentes etapas de desarrollo. Ellos concluyen que los mejores criterios de efectividad son importantes en cada uno de las etapas del ciclo de vida organizacional.

Existen relaciones estrechas entre las etapas de desarrollo en los ciclos de vida de la organización y los cuatro tipos de cultura en la del marco de valores competitivos, Quinn y Cameron (1983) revisaron nueve modelos de ciclos de vida organizacional que se habían propuesto en la literatura y derivaron un modelo resumido de etapas del ciclo de vida que integra cada uno de los nueve modelos. Este modelo resumido del ciclo de vida incluye cuatro etapas: etapa emprendedora, etapa de colectividad, etapa de formalización y control y elaboración de la etapa de estructura. Sobre la base de ciertas características que caracterizan a las organizaciones en diferentes 
etapas de desarrollo, plantearon la hipótesis de que ciertos criterios de eficacia en el Marco de Valores Competitivos (CVF) son importantes en determinadas etapas del ciclo de vida, pero no en otros. También concluyeron que los principales criterios de eficacia (por lo tanto, con los principales tipos de cultura organizacional - los autores) cambian de manera predecible a medida que las organizaciones se desarrollan a lo largo de sus ciclos de vida. En la etapa empresarial, caracterizada por la innovación, la creatividad y la organización de los recursos, el tipo de cultura más fuerte parece ser la cultura en si. Las organizaciones en la etapa de colectividad parecen estar caracterizadas por la comunicación y la estructura informales, el sentido de la familia y la cooperación entre los miembros, el alto compromiso de los miembros y el liderazgo personalizado, que están asociados con la cultura del clan. En la etapa de formalización, la estabilidad organizacional, la eficiencia de la producción, las reglas y procedimientos, y las tendencias conservadoras que tipifican las organizaciones (Deshpande \& Farley, 2004).

Los tipos de cultura parecen ser principalmente la cultura jerárquica y la cultura del mercado. En la cuarta etapa, elaboración de estructura, la organización monitorea el entorno externo para renovarse o expandir su dominio. La cultura parece recibir el mayor énfasis en esta etapa. Un estudio de caso longitudinal realizado por Quinn y Cameron (1983) proporcionó una cierta evidencia para apoyar las relaciones hipotéticas entre las etapas del ciclo de vida y los tipos de la cultura, que demostraron el potencial del modelo en diagnosticar y predecir cambio de la cultura de organización, sobre todo el que se encuentra en base a la cultura interna (Endomarketing) y en lo relacionado a los procesos internos de calidad.

\section{Metodología}

El Instrumento de Faedpyme se aplicó en la parte sureste del Estado de Coahuila en el País de México. En su tropicalización se obtuvo un alfa de Cronbach's de .81 lo que señala que tiene confiabilidad. El tamaño muestral se fundamentó en los principios del muestreo estratificado, primero con estratos del tipo giro al que se dedican industria, construcción, comercio y servicios de las diferentes miPyme's que se encuestaron en México. Se seleccionaron las empresas del giro empresarial, Estas empresas tenían al menos 5 a 10 trabajadores, de 11 a 50 trabajadores y de 51 a 250 trabajadores, se encuestaron 54 empresas del giro industrial. Se utilizaron ocho variables del constructo de rendimiento éstas se basan en una aproximación a la clasificación propuesta por Quinn y Rohrbaugh. (1983). Para valorar las variables se utiliza una escala de 1 a 5 en donde $1=$ "total desacuerdo" y 5 = "total acuerdo". 
El cuestionario de Faedpyme, que incluye una tipología de preguntas muy variada, tanto cuantitativas como cualitativas, el constructo que se factoriza tiene escala. Para el análisis se aplicó el Análisis Factorial Exploratorio, que obtuvo un KMO de .851 y un Test de Bartlett (1999) con alfa de 0.01; y de acuerdo con el muestreo establecido por Faedpyme el instrumento fue aplicado con un error que no superase los tres puntos porcentuales y con un nivel de confianza al 95\% (Briones, 1992).

\section{Resultados}

Se realiza un análisis factorial para conocer la descomposición del constructo rendimiento. Con extracción de principales componentes, Método de Rotación Varimax, con 21 convergencias, y considerando solo valores mayores de 0.30 de acuerdo con Hair (1999).

Tabla 1. Matriz de componente rotado ${ }^{\mathrm{a}, \mathrm{b}}$

\begin{tabular}{ccc}
\hline \hline & \multicolumn{2}{c}{ Componente } \\
\cline { 2 - 3 } Variables & 1 & 2 \\
\hline Dispone de procesos internos más eficientes & .851 \\
Tiene empleados más satisfechos/motivados & .805 \\
Tiene menor ausentismo laboral & .714 & \\
Está creciendo más & .681 & .894 \\
Es más rentable & .609 & .834 \\
Ofrece Productos de Mayor calidad & .724 \\
Cuenta con clientes más satisfechos & \\
Se adapta antes a los cambios del mercado & \\
\hline \hline Método de extracción: análisis de componentes principales. \\
Método de rotación: Varimax con normalización Kaiser. \\
a. La rotación ha convergido en 3 iteraciones. \\
b. Sólo se utilizan los casos para los cuales Sector = 1 Industria en la fase de análisis.
\end{tabular}

\section{Fuente Elaboración propia.}

En la Tabla 1 se muestran los resultados de Análisis Factorial Exploratorio, para las Pymes de giro industrial, en la región sur del Estado de Coahuila México, encontrándose solo dos componentes, de los cuales el primer es llamado Endomarketing debido a sus principales componentes son variables en las que se encuentran relacionados los indicadores en las que el personal tiene un papel importante como lo son empleados más motivados, que son los que arrojan procesos más eficientes; los empleados están más satisfechos y por lo tanto presentan el comportamiento de menor ausentismo laboral, lo que probablemente tenga como consecuencia más crecimiento empresarial además esto vuelve la empresa más rentable en su giro empresarial. En el segundo componente o factor, se encuentra que están relacionados los indicadores de adaptación a los cambios de mercado, cuenta con clientes más satisfechos y ofrece productos de mayor calidad. Lo anterior es posiblemente una consecuencia de que los empleados al estar 
estableciendo Endomarketing obtengan como resultado las mejoras de la producción, y ofrecimiento de productos de más calidad que satisfacen al cliente en el ámbito empresarial.

\section{Conclusion}

Las diferencias de posición de las empresas con respecto a la competencia se analizan considerando el sector. Las empresas del sector industrial, presentan una evolución más favorable que las micro y pequeñas empresas, especialmente en el incremento de la rentabilidad, el crecimiento de su nivel de ventas y la disponibilidad de procesos internos más eficientes.

Se considera que los resultados del presente trabajo muestran elementos necesarios para determinar que en las miPyme's, del giro empresarial, la aplicación con cierto conocimiento de las prácticas de la mercadotecnia, como lo es el Endomarketing, han empezado a dar sus frutos al reducir uno de los principales problemas de las empresas industriales, que es el alto índice de rotación, y que cuando se empiezan a conocer técnicas de marketing que se pueden aplicar a este giro empresarial, los resultados tienen consecuencias de mejoras de producción y menores índices de faltas del personal obteniendo también satisfacción de los empleados y consecuentemente producción con más calidad que mantiene una clientela satisfecha.

\section{References:}

1. Briones, G. (1992). Métodos y Técnicas de Investigación para las Ciencias Sociales. México: Trillas.

2. Deshpande, R., \& Farley, J. U. (2004). Organizational Culture, Market Orientation Innovativeness and Firm Performance: An international Research Odyssey. Journal of Research in Marketing, 12(3), 3-22.

3. García Pérez de Lerma , D., Martínez García , F. J., \& Aragón Sánchez , A. (2011). FAEDPYME. Universidad Politécnica de Cartagena.

4. Hair, J. F., E., A. R., Tatham, R. L., \& Black, W. C. (1999). Análisis Multivariante (quinta Edición). Madrid, España.: Pearson Educación.

5. Hernández, S. R., Fernández, C. C., \& Baptista, L. P. (2014). Metodología de la Investigación (Sexta Edición). México: McGrawHill.

6. Lund, B. (2003). Organizational Culture and Job Satisfaction. Journal of Business and Industrial Marketing, 18(3), 219-236.

7. Nunnally, J. C. (1987). Teoría Psicométrica. México: Ed. Trillas . 
8. Quinn, R. E., \& Rohrbaugh, J. (1983). A Spatial Model of Effectiveness Criterio: some preliminary evidencia. Management Sciencie, 29(1), 33-51. 